\title{
Single MO-CCCCTA-Based Electronically Tunable Current/Trans-Impedance-Mode Biquad Universal Filter
}

\author{
Sajai Vir Singh ${ }^{1}$, Sudhanshu Maheshwari ${ }^{2}$, Durg Singh Chauhan ${ }^{3}$ \\ ${ }^{1}$ Department of Electronics and Communications, Jaypee University of Information Technology, \\ Waknaghat, India \\ ${ }^{2}$ Department of Electronics Engineering, Zakir Hussain College of Engineering and Technology, \\ Aligarh Muslim University, Aligarh, India \\ ${ }^{3}$ Department of Electrical Engineering, Institute of Technology, Banaras Hindu University, Varanasi, India \\ E-mail:sajaivir@rediffmail.com,maheshwarispm@rediffmail.com,pdschauhan@gmail.com \\ Received June 12, 2010; revised July 9, 2010; accepted July 19, 2010
}

\begin{abstract}
This paper presents an electronically tunable current/trans-impedance-mode biquad universal filter employing only single multi-output current controlled current conveyor trans-conductance amplifier (MO-CCCCTA) and two grounded capacitors. The proposed filter realizes all the standard filter functions i.e. low pass (LP), band pass (BP) and high pass (HP), notch and all pass (AP) filters in the current form at high impedance output through appropriate selection of the input signals, without any matching conditions. Simultaneously, it can also realize all the standard filter functions in trans-impedance form from the same circuit topology. The circuit does not require inverting-type input current signal(s) and double input current signal(s) to realize all the responses in the design. The validity of proposed filter is verified through PSPICE simulations.
\end{abstract}

Keywords: Universal, Current-Mode, Trans-Impedance-Mode, Biquad Filter

\section{Introduction}

Analog electronic filters are important blocks, widely employed in continuous time signal processing. They are present in just about every piece of electronic equipment that are obvious types of equipments, such as radios, televisions and stereo systems. Test equipments such as spectrum analyzers and signal generators also need filters even where signals are connected into digital form, using digital to analog converters; analog filters are usually needed to prevent aliasing. Universal biquadratic filters belong to most popular analog filters, providing all standard filter functions (LP, BP, HP, Notch and AP), without modifying the circuit topology. Several filter realizations either in current-mode, where the input and the output variables are current, or in voltage-mode, where the input and output variables are voltage, have been reported using different active elements [1-22]. These filter circuits are classified as single input multiple output (SIMO) [1-10], multiple input single output (MISO) [11-19] and multiple input multiple output (MIMO) [20-22]. However, there are a number of ap- plications in analog signal processing where it may be desirable to have active filters with input variable as current and output variable as voltage that is transimpedance filters. Such filters can be used as an interface circuit connecting a current-mode circuit to a voltage-mode circuit and find direct applications with some sensors, the receiver base band (BB) blocks of modern radio systems and $\mathrm{D} / \mathrm{A}$ converters which provide a current as output signal, avoiding a current to voltage conversion $[23,24]$. There are a small number of filter topologies operating in trans-impedance-mode reported in the literature [25-27]. These filter topologies reported in [24-26] cannot realize all the standard filter functions (LP, BP, HP, Notch and AP). As far as the topic of this paper is concerned, the filter circuits operated in either current-mode or trans-impedance-mode or in both modes simultaneously, using a single active element, are of interest. Single active element based current-mode filters with multi-input are reported in [18-20]. The circuits in references $[18,19]$ use three inputs and one output and realize all the standard filter functions at high impedance output terminal. The filter circuit of [18] 
employs single CCCII, two grounded capacitors and one floating resistor and suffers from the following disadvantages: 1) requirement of passive component matching conditions, 2) requirement of inverting-type input current signal, 3) use of floating resistor which is not suitable for IC fabrications while other filter circuit of [19] uses single CCCCTA, two grounded capacitors and suffers from the following two disadvantages: requirement of double input current signal to obtain an all-pass response and use of one capacitor at port $\mathrm{X}$ which limits the use of filter in high frequency range since it effectively appears in series with $X$ terminal resistance [28]. Lastly, a three inputs and two outputs current-mode single DO-CCCDTA based filter circuit [20] also realizes all the standard filter functions at high impedance outputs but it still require double input current signal to obtain an all-pass response. Up until now, no previous paper has reported a filter based on single active element which can realize all the standard responses in current as well as trans-impedance form, together, without any matching conditions, from the same topology. In this paper a single MO-CCCCTAbased electronically tunable current/trans-impedancemode biquad universal filter is proposed. It also uses two grounded capacitors. The proposed filter realizes all the standard filter functions i.e. LP, BP, HP, notch and AP filters in the current form at high impedance output through appropriate selection of the input signals, without any matching conditions. Simultaneously, it can also realize all the standard filter functions in trans-impedance form from the same circuit topology. The proposed circuit does not require inverting-type input current signal(s) and double input current signal(s) to realize all the responses in the design. The proposed circuit does not use capacitor at port $X$ so this circuit is suitable in high frequency range. The circuit possesses low active and passive sensitivity. Moreover, the pole frequency $\left(\omega_{o}\right)$ can be independently tuned without disturbing the parameter $\omega_{o} / Q$ through adjusting the bias current of MO-CCCCTA. The performance of proposed circuit is illustrated by PSPICE simulation using $0.35 \mu$ CMOS parameters.

\section{Proposed Circuit}

CCCCTA is relatively new proposed current mode active building block [19] which is the modified version of CCTA. This device can be operated in both current and voltage modes, providing flexibility. In addition, it can offer several advantages such as high slew rate, high speed, wider bandwidth and simpler implementation. Moreover, in the CCCCTA one can control the parasitic resistance at $X\left(R_{X}\right)$ port by input bias current. The MO-CCCCTA properties can be described in the following matrix equation

$$
\left[\begin{array}{c}
I_{Y} \\
V_{X} \\
I_{-Z a,-Z c} \\
I_{Z b} \\
I_{-O b} \\
I_{-O c}
\end{array}\right]=\left[\begin{array}{cccccc}
0 & 0 & 0 & 0 & 0 & 0 \\
R_{x} & 1 & 0 & 0 & 0 & 0 \\
-1 & 0 & 0 & 0 & 0 & 0 \\
1 & 0 & 0 & 0 & 0 & 0 \\
0 & 0 & -g_{m b} & 0 & 0 & 0 \\
0 & 0 & 0 & -g_{m c} & 0 & 0
\end{array}\right]\left[\begin{array}{c}
I_{X} \\
V_{Y} \\
V_{Z b} \\
V_{-Z c} \\
V_{-O b} \\
V_{-O c}
\end{array}\right]
$$

where $R_{X}$ is the parasitic resistance at $X$ terminal. $g_{m b}$ and $g_{m c}$ are trans-conductance of CCCCTA. The schematic symbol of MO-CCCCTA is illustrated in Figure 1. CMOS implementation of MO-CCCCTA is shown in Figure 2. For a CMOS CCCCTA [29], the $R_{X}, g_{m b}$ and $g_{m c}$ can be expressed to be

$$
R_{X}=\frac{1}{\sqrt{8 \beta_{n} I_{B}}}, g_{m b}=\sqrt{\beta_{n} I_{S b}} \text { and } g_{m c}=\sqrt{\beta_{n} I_{S c}}
$$

where

$$
\beta_{n}=\mu_{n} C_{O X} \frac{W}{L}
$$

where $\mu_{n}, C_{O X}$ and $W / L$ are the electron mobility, gate oxide capacitance per unit area and transistor aspect ratio, respectively. $I_{B}, I_{S b}$ and $I_{S c}$ are the biasing currents of MO-CCCCTA.

The proposed biquad filter circuit as shown in Figure 3 uses only single MO-CCCCTA and two grounded capacitors. By routine analysis of the circuit in Figure 3, the output current $I_{O}$ and output voltage $V_{O}$ can be obtained as

$$
\begin{gathered}
I_{O}=\frac{I_{1} s^{2} C_{1} C_{2}-I_{2} s C_{2} g_{m c}+I_{3} g_{m b} g_{m c}}{s^{2} C_{1} C_{2}+s C_{2} g_{m c}+g_{m b} g_{m c}} \\
V_{O}=\frac{R_{x}\left(I_{1} s^{2} C_{1} C_{2}-I_{2} s C_{2} g_{m c}+I_{3} g_{m b} g_{m c}\right)}{s^{2} C_{1} C_{2}+s C_{2} g_{m c}+g_{m b} g_{m c}}
\end{gathered}
$$

From Equations (4) and (5), various filter responses in current form as well as in trans-impedance form can be

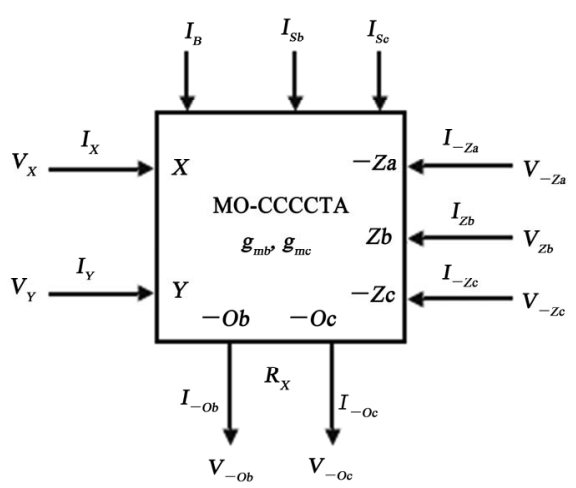

Figure 1. MO-CCCCTA symbol. 


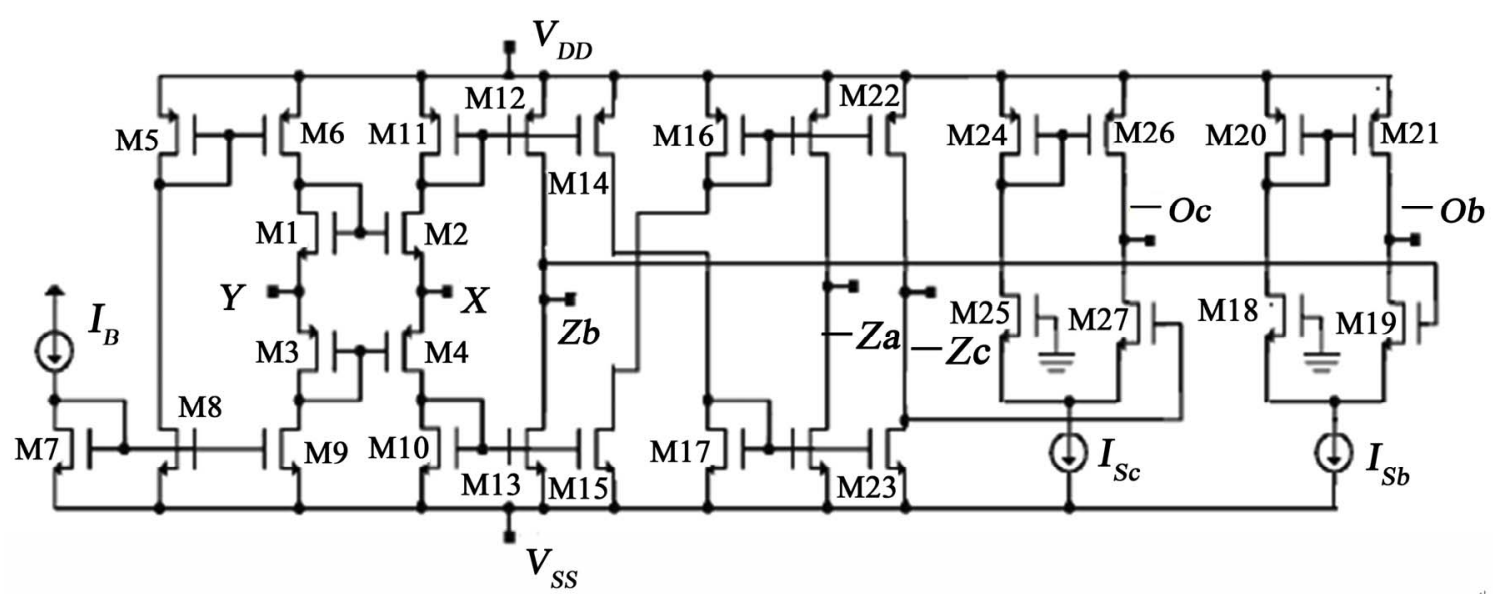

Figure 2. CMOS implementation of MO-CCCCTA.

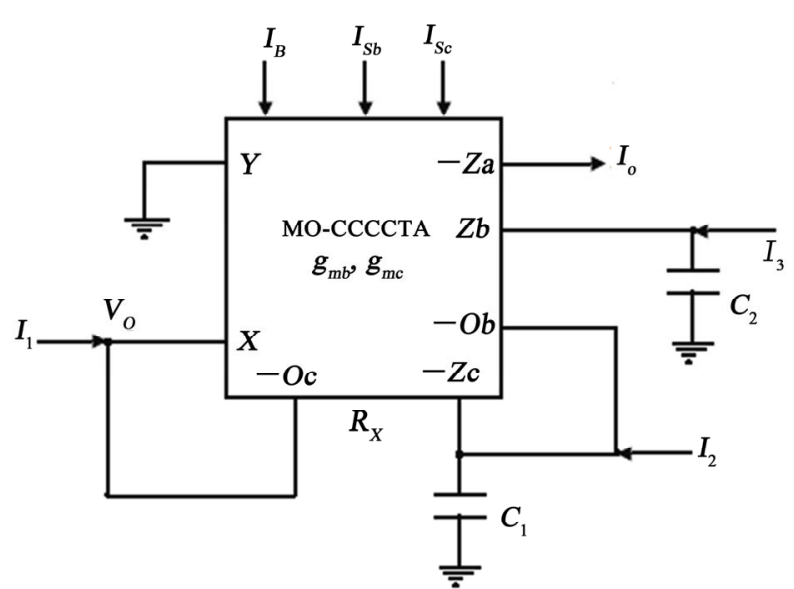

Figure 3. proposed current/trans-impedance-mode universal filter.

obtained through appropriate selection of input currents.

1) High pass response in current form as well as in trans-impedance form, with $I_{1}=I_{i n}, I_{2}=I_{3}=0$.

2) Low pass response in current form as well as in trans-impedance form, with $I_{1}=I_{2}=0, I_{3}=I_{i n \text {. }}$.

3) Inverted band pass response in current form as well as in trans-impedance form, with $I_{1}=I_{3}=0, I_{2}=I_{i n \text {. }}$.

4) Notch response in current form as well as in trans-impedance form, with $I_{1}=I_{3}=I_{i n}, \quad I_{2}=0$.

5) All pass response in current form as well as in trans-impedance form, with $I_{1}=I_{2}=I_{3}=I_{i n}$.

Thus, the circuit is capable of realizing all the standard filter responses in current as well as in trans-impedancemode from the same configuration, without any matching constraints. Moreover, there is no requirement of inverting-type input current signal(s) and double input current signal(s) to realize all the responses in the design.

The filter parameters pole frequency $\left(\omega_{o}\right)$, the quality factor $(Q)$ and bandwidth $(B W) \omega_{o} / Q$ can be expressed as

$$
\begin{gathered}
\omega_{o}=\left(\frac{g_{m c} g_{m b}}{C_{1} C_{2}}\right)^{\frac{1}{2}}=\left(\frac{1}{C_{1} C_{2}} \beta_{n}\right)^{\frac{1}{2}}\left(I_{S c} I_{S b}\right)^{\frac{1}{4}}, \\
Q=\left(\frac{C_{1} g_{m b}}{C_{2} g_{m c}}\right)^{\frac{1}{2}}=\left(\frac{C_{1}}{C_{2}}\right)^{\frac{1}{2}}\left(\frac{I_{S b}}{I_{S c}}\right)^{\frac{1}{4}} \\
B W=\frac{\omega_{O}}{Q}=\frac{g_{m c}}{C_{1}}=\frac{1}{C_{1}}\left(\beta_{n} I_{S c}\right)^{\frac{1}{2}}
\end{gathered}
$$

From (6) by maintaining the ratio $I_{S b}$ and $I_{S c}$ to be constant, it can be remarked that the pole frequency can be adjusted by $I_{S b}$ and $I_{S c}$ without affecting the quality factor. In addition, pole frequency can be controlled by $I_{S b}$ without affecting bandwidth $(B W)$ of the system. To see the effects of non idealities, the defining equations of the MO-CCCCTA can be rewritten as the following.

$$
\begin{gathered}
V_{X}=\beta V_{Y}+I_{X} R_{X}, I_{-Z a}=-\alpha_{a} I_{X}, I_{Z b}=\alpha_{b} I_{X} \\
I_{-Z c}=-\alpha_{c} I_{X}, I_{-O b}=-\gamma_{b} g_{m b} V_{Z b}, I_{-O c}=-\gamma_{c} g_{m c} V_{-Z c}
\end{gathered}
$$

where $\beta, \alpha_{a}, \alpha_{b}, \alpha_{c}, \gamma_{b}$ and $\gamma_{c}$ are transferred error values deviated from one. In the case of non-ideal and re-analyzing the proposed filter in Figure 3, it yields the current output and voltage output as

$$
\begin{aligned}
& I_{O}=\frac{\alpha_{a}\left(I_{1} s^{2} C_{1} C_{2}-I_{2} s \gamma_{c} C_{2} g_{m c}+I_{3} \gamma_{b} \gamma_{c} g_{m b} g_{m c}\right)}{s^{2} C_{1} C_{2}+s \gamma_{c} \alpha_{c} C_{2} g_{m c}+\gamma_{b} \gamma_{c} \alpha_{b} g_{m b} g_{m c}} \\
& V_{O}=\frac{\alpha_{a} R_{X}\left(I_{1} s^{2} C_{1} C_{2}-I_{2} s \gamma_{c} C_{2} g_{m c}+I_{3} \gamma_{b} \gamma_{c} g_{m b} g_{m c}\right)}{s^{2} C_{1} C_{2}+s \gamma_{c} \alpha_{c} C_{2} g_{m c}+\gamma_{b} \gamma_{c} \alpha_{b} g_{m b} g_{m c}}
\end{aligned}
$$

In this case, the $\omega_{o}$ and $Q$ are changed to

$$
\omega_{o}=\left(\frac{\gamma_{b} \gamma_{c} \alpha_{b} g_{m b} g_{m c}}{C_{1} C_{2}}\right)^{\frac{1}{2}}, Q=\frac{1}{\alpha_{c}}\left(\frac{\gamma_{b} \alpha_{b} C_{1} g_{m b}}{\gamma_{c} C_{2} g_{m c}}\right)^{\frac{1}{2}}
$$

The all active and passive sensitivities can be found as 


$$
\begin{gathered}
S_{C_{1}, C_{2}}^{\omega_{o}}=-\frac{1}{2}, S_{\gamma_{b}, \gamma_{c}, \alpha_{b}, g_{m b}, g_{m c}}^{\omega_{o}}=\frac{1}{2}, S_{\alpha_{a}, \alpha_{c}, \beta, R_{X}}^{\omega_{o}}=0 \\
S_{C_{2}, g_{m c}, \gamma_{c}}^{Q}=-\frac{1}{2}, S_{C_{1}, \alpha_{b}, \gamma_{b}, g_{m b}}^{Q}=\frac{1}{2}, S_{\alpha_{c}}^{Q}=-1 \quad S_{\alpha_{a}, \beta, R_{X}}^{Q}=0
\end{gathered}
$$

From the above results, it can be observed that all the active and passive sensitivities are equal or less than 1 in magnitude.

\section{Simulation Results}

The PSPICE simulations are carried out to demonstrate the feasibility of the proposed circuit using CMOS implementation as shown in Figure 2. The simulations use a $0.35 \mu \mathrm{m}$ MOSFET [30] from TSMC. The dimensions of PMOS are determined as $W=3 \mu \mathrm{m}$ and $L=2 \mu \mathrm{m}$. In NMOS transistors, the dimensions are $W=3 \mu \mathrm{m}$ and $L$ $=4 \mu \mathrm{m}$. The circuit is designed for $Q=1$ and $f_{o}=\omega_{o} /$ $2 \pi=1.57 \mathrm{MHz}$. The active and passive components are chosen as $I_{B}=7.5 \mu \mathrm{A}, I_{S b}=I_{S c}=30.65 \mu \mathrm{A}$ and $C_{I}=C_{2}=$ $7.5 \mathrm{pF}$. Figure 4 Shows the simulated gain and phase responses of the HP, LP, BP, Notch and AP in the current

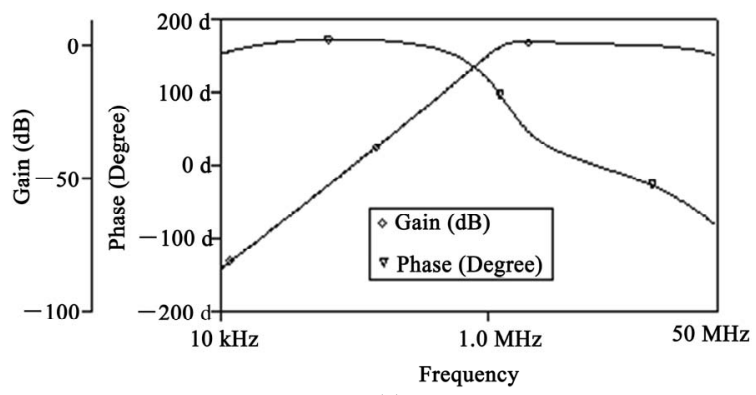

(a)

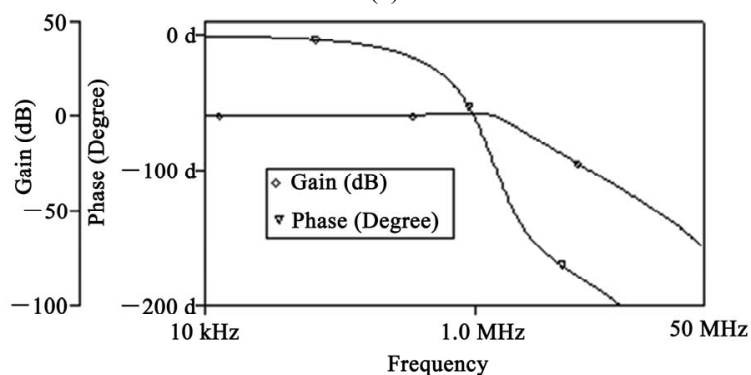

(b)

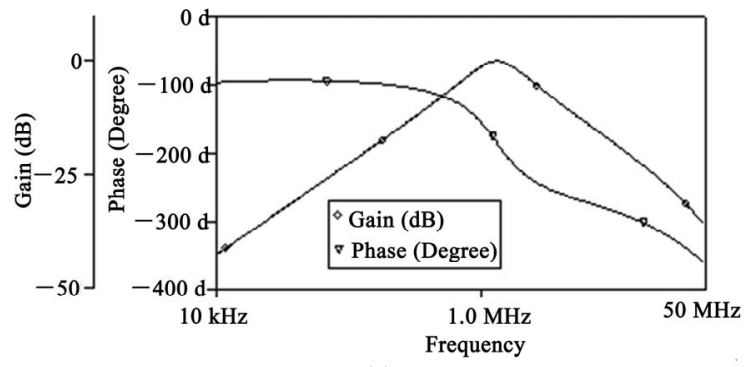

(c)

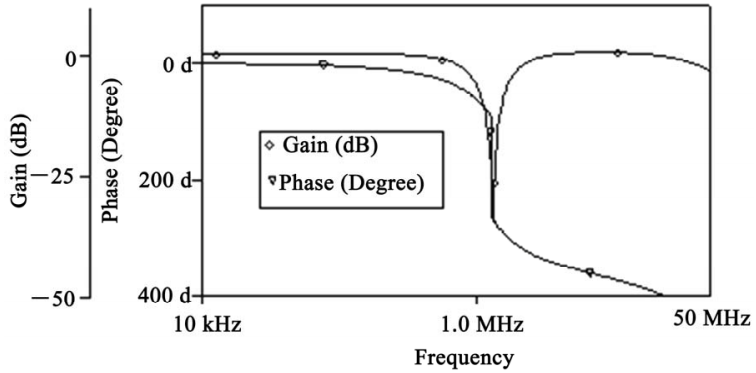

(d)

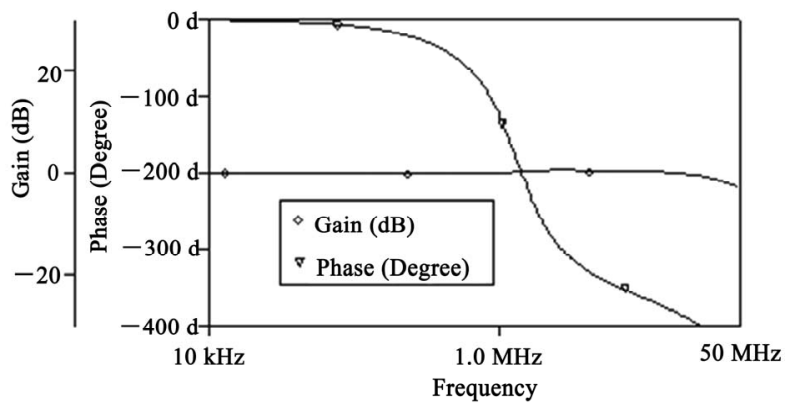

(e)

Figure 4. Current gain and Phase responses of the proposed filter (a) HP, (b) LP, (c) BP, (d) Notch and (e) AP.

form, of the proposed circuit in Figure 3. The supply voltages are $V_{D D}=-V_{S S}=2.5 \mathrm{~V}$. The simulated pole frequency is obtained as $1.35 \mathrm{MHz}$. It is noted that simulation results agree quite well with theoretical ones as expected, whereas the difference between them arises from non-idealities such as non ideal gain and parasitic elements. The power dissipations of the proposed circuit for the design values is found as $0.629 \mathrm{~mW}$ that is a low value.

Next, the frequency tuning aspect of the circuit is verified for a constant $Q(=1)$ value for the BP response in current-mode. The bias currents $I_{S b}$ and $I_{S c}$ are varied simultaneously, by keeping its ratio to be constant. The pole frequency variation, for $Q=1$, is shown in Figure 5. The frequency is found to vary as $650 \mathrm{kHz}, 990 \mathrm{kHz}$, $1.34 \mathrm{MHz}$ and $1.8 \mathrm{MHz}$ for four values of $I_{S b}=I_{S c}=6 \mu \mathrm{A}$, $15 \mu \mathrm{A}, 30 \mu \mathrm{A}$ and $60 \mu \mathrm{A}$, respectively. Further simulations are carried out to verify the total harmonic distortion (THD). The circuit is simulated for THD analysis at BP output in current-mode, by applying sinusoidal input current of varying amplitude and constant frequency. The THD values for the input current signal having amplitude less than $40 \mu \mathrm{A}$, at frequency $1.35 \mathrm{MHz}$ remain in acceptable limits i.e. 4\%. The time domain response of band-pass output in current form is shown in Figure 6. It is observed that $40 \mu \mathrm{A}$ peak to peak input current sinusoidal signal levels are possible without significant distortions. Thus both THD analysis and time domain response of BP output in current-mode confirm the practical utility of the proposed circuit. 


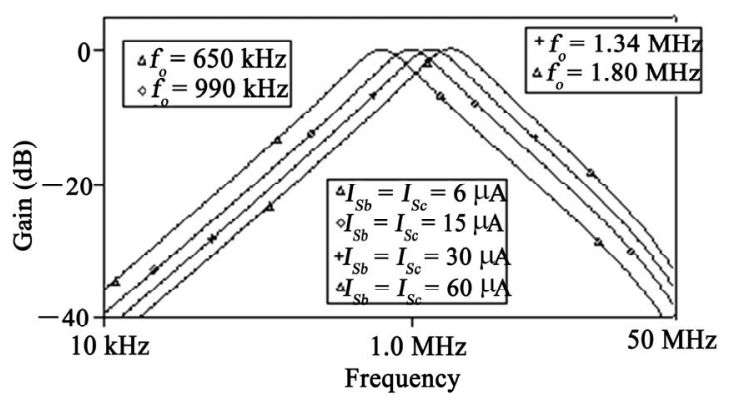

Figure 5. Band pass responses in current-mode for different values of $I_{S b}=I_{S c}$ of the proposed filter.

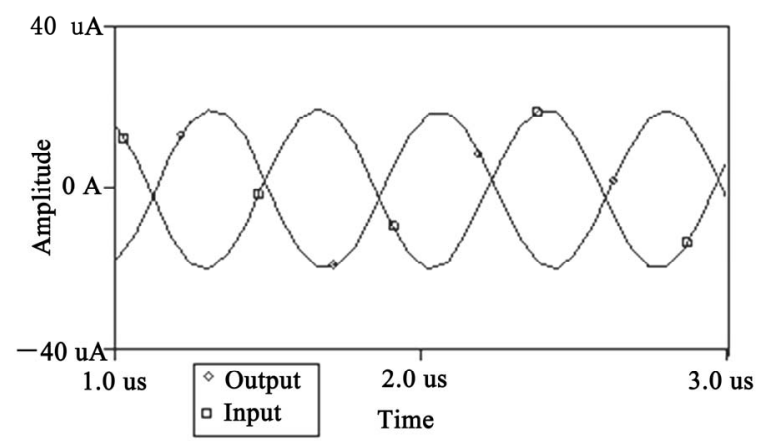

Figure 6. The sinusoidal input having frequency of $1.35 \mathrm{MHz}$ and corresponding band pass output waveforms in current-mode of the proposed filter.

\section{Conclusions}

This paper presents an electronically tunable current/ trans-impedance-mode biquad universal filter using single MO-CCCCTA. The proposed filter offers the following advantages: 1) realization of LP, HP, BP, Notch and AP responses in current form as well as in trans-impedance form without changing the circuit topology; 2) both the capacitors being permanently grounded; 3 ) low sensitivity figures, low THD and low power consumptions; 4) independent current control of $\omega_{o}$ without disturbing $\left.\omega_{o} / Q ; 5\right)$ no requirement of components matching conditions to get all filter responses; 6) no requirements of inverting-type input current signal(s) and double input current signal(s) to realize the response(s) in the design; 7) single active element.

\section{References}

[1] S. Minaei and S. Türköz, "New Current-Mode Current-Controlled Universal Filter with Single Input and Three Outputs," International Journal of Electronics, Vol. 88, No. 3, 2001, pp. 333-337. doi:10.1080/00207210010 013201

[2] T. Katoh, T. Tsukutani, Y. Sumi and Y. Fukui, "Electronically Tunable Current-Mode Universal Filter Employing CCCIIs and Grounded Capacitors," International
Symposium on Intelligent Signal Processing and Communications, 2006, pp. 107-110.

[3] S. Minaei and S. Türköz, "Current-Mode Electronically Tunable Universal Filter Using Only Plus-Type Current Controlled Conveyors and Grounded Capacitors," Electronics and Telecommunication Research Institute Journal, Vol. 26, No. 4, 2004, pp. 292-296. doi:10.4218/etri j.04.0103.0146

[4] I. A. Khan and M. H. Zaidi, "Multifunction Translinear-C Current Mode Filter," International Journal of Electronics, Vol. 87, No. 6, 2000, pp. 1047-1051. doi:10.1080/002 072100412984

[5] M. Soliman, "Current Mode Universal Filters Using Current Conveyors," Circuits Systems and Signal Processing, Vol. 27, No. 3, 2008, pp. 405-427. doi:10.1007/s0003 4-008-9034-y

[6] T. Tsukutani, Y. Sumi, S. Iwanari and Y. Fukui, "Novel Current-Mode Biquad Using MO-CCCIIs and Grounded Capacitors," Proceedings of 2005 International Symposium on Intelligent Signal Processing and Communication Systems, 13-16 December 2005, pp. 433-436.

[7] D. R. Bhaskar, A. K. Singh, A. K. Sharma and R. Senani, "New OTA-C Universal Current-Mode/Trans-Admittance Biquads," Institute of Electronics, Information and Communication Engineering Electronics Express, Vol. 2, No. 1, 2005, pp. 8-13. doi:10.1587/elex.2.8

[8] J. Wu and E. I. El-Masry, "Universal Voltage and Current-Mode OTAs Based Biquads," International Journal of Electronics, Vol. 85, No. 5, 1998, pp. 553-560. doi: 10.1080/002072198134355

[9] C. M. Chang and M. J. Lee, "Voltage-Mode Multifunction Filter with Single Input and Three Outputs Using Two Compound Current Conveyors," IEEE Transactions on Circuits and Systems- I: Fundamental Theory and Applications, Vol. 46, No. 11, 1999, pp. 1364-1365. doi:10. 1109/81.802827

[10] S. Maheshwari, "Analogue Signal Processing Applications Using a New Circuit Topology," IET Circuits, Devices and Systems, Vol. 3, No. 3, 2009, pp. 106-115. doi: 10.1049/iet-cds.2008.0294

[11] T. Praveen, M. T. Ahmed and I. A. Khan, "A Canonical Voltage-Mode Universal CCCII-C Filter," Journal of Active and Passive Devices, Vol. 4, No. 1-2, 2009, pp. 7-12.

[12] S. Maheshwari, "High Performance Voltage-Mode Multifunction Filter with Minimum Component Count," WSEAS Transactions on Electronics, Vol. 5, No. 6, 2008, pp. 244-249.

[13] M. T. Abuelma'atti and N. A. Tassaduq, "A Novel Three Inputs and One Output Universal Current-Mode Filter Using Plus-Type CCIIs," Microelectronics Journal, Vol 30, No. 3, 1999, pp. 287-292. doi:10.1016/S0026-2692 (98)00169-4

[14] R. Senani, "New Universal Current Mode Biquad Employing All Grounded Passive Components but Only Two DOCCs," Journal of Active and Passive Devices, Vol. 1, No. 3-4, 2006, pp. 281-288.

[15] M. T. Abuelma'atti and M. L. Al-qahtani, "Universal 
Current-Contolled Current-Mode Filter with Three Inputs and One Output Using Current Controlled Conveyor," Active Passive Electronics Components, Vol. 21, No. 1, 1998, pp. 33-41. doi:10.1155/1998/83413

[16] W. Tangsriart and W. Surakampontorm, "Electronically Tunable Current-Mode Universal Filter Employing Only Plus-Type Current-Controlled Conveyors and Grounded Capacitors," Circuits Systems and Signal Processing, Vol. 25, No. 6, 2006, pp. 701-713. doi:10.1007/s00034-005-0 $908-\mathrm{y}$

[17] W. Tangsrirat and W. Surakampontorm, "Low-Component Current-Mode Universal Filter Using Current-Controlled Conveyors and Grounded Capacitors," Journal of Active and Passive Devices, Vol. 4, No. 3, 2009, pp. 259-264.

[18] C. M. Chang, "Universal Active Current Filter Using Single Second-Generation Current Controlled Conveyor," International Journal of Circuits, Systems and Signal Processing, Vol. 1, No. 1, 2007, pp. 194-198.

[19] M. Siripruchyanun and W. Jaikla, "Current Controlled Current Conveyor Transconductance Amplifier (CCCCTA): A Building Block for Analog Signal Processing," Electrical Engineering, Vol. 90, No. 6, 2008, pp. 443-453. doi:10.1007/s00202-007-0095-x

[20] M. Siripruchyanun and W. Jaikla, "Electronically Controllable Current-Mode Universal Biquad Filter Using Single DO-CCCDTA," Circuits System Signal Processing, Vol. 27, No. 1, 2008, pp. 113-122. doi:10.1007/s00034-0 08-9014-2

[21] W. Tangsriart, "Current-Tunable Current-Mode Multifunction Filter Based on Dual-Output Current-Controlled Conveyors," International Journal of Electronics and Communications, Vol. 61, No. 8, 2007, pp. 528-533. doi:10.1016/j.aeue.2006.09.005

[22] T. Tsukutani, Y. Sumi and N. Yabuki, "Versatile Current Mode Biquadratic Circuit Using Only Plus Type CCCIIs and Grounded Capacitors," International Journal of Electronics, Vol. 94, No. 12, 2007, pp.1147-1156. doi:10. 1080/00207210701789998
[23] Y. Wang and R. Raut, "A Design of Transresistance Amplifier for High Gain-Bandwidth Applications," Proceedings of the 2003 10th IEEE International Conference on Electronics, Circuits and Systems, 14-17 December 2003, pp. 185-188.

[24] A. Carlosena and E. Cabral, "Novel Transimpedance Filter Topology for Instrumentation," IEEE Transactions on Instrumentation and Measurement, Vol. 46, No. 4, 1997, pp. 862-867. doi:10.1109/19.650789

[25] M. Soliman, "Mixed-Mode Biquad Circuits," Microelectronics Journal, Vol. 27, No. 6, 1996, pp. 591-594. doi:10. 1016/0026-2692(95)00121-2

[26] J. Ramirez-Angulo, M. Robinson and E. Sanchez-Sinencio, "Current-Mode Continuous-Time Filters: Two Design Approaches," IEEE Transactions on Circuits and Systems-II: Analog and Digital Signal Processing, Vol. 39, No. 6, 1992, pp. 337-341. doi:10.1109/82.145290

[27] S. Minaei, G. Topcu and O. Cicekoglu, "Low Input Impedance Trans-Impedance Type Multifunction Filter Using Only Active Elements," International Journal of Electronics, Vol. 92, No. 7, 2005, pp. 385-392. doi:10. 1080/00207210412331334789

[28] A. Fabre, O. Saiid and H. Barthelemy, "On the Frequency Limitations of the Circuits Based on Second Generation Current Conveyors," Analog Integrated Circuits and Signal Processing, Vol. 7, No. 2, 1995, pp. 113-129. doi:1 0.1007/BF01239166

[29] M. Siripruchyanun, P. Silapan and W. Jaikla, "Realization of CMOS Current Controlled Current Conveyor Transconductance Amplifier and Its Application," Journal of Active and Passive Devices, Vol. 4, No. 1-2, 2009, pp. 35-53.

[30] E. Yuce, S. Tokar, A. Kizilkaya and O. Cicekoglu, "CCII-Based PID Controllers Employing Grounded Passive Componensts," International Journal of Electronics and Communications, Vol. 60, No. 5, 2006, pp. 399-403. doi:10.1016/j.aeue.2005.03.017 